\title{
The Dynamics of Implicit Transactions Costs
}

\author{
Ming Guo \\ ShanghaiTech University, Shanghai, China \\ Email: guoming@shanghaitech.edu.cn
}

How to cite this paper: Guo, M. (2020) The Dynamics of Implicit Transactions Costs. Journal of Mathematical Finance, 10, 705-716.

https://doi.org/10.4236/jmf.2020.104041

Received: July 23, 2020

Accepted: November 24, 2020

Published: November 27, 2020

Copyright $\odot 2020$ by author(s) and Scientific Research Publishing Inc. This work is licensed under the Creative Commons Attribution International License (CC BY 4.0).

http://creativecommons.org/licenses/by/4.0/

\begin{abstract}
We use tick-by-tick data to study the dynamics of different components of implicit trading costs, including market impact cost, proportional cost, and fixed cost, over a decade. We model the proportional cost and the fixed cost as compensation for an affine structure of trading costs faced by the market maker. We find that the market impact cost remains stable over time, whereas the fixed cost and the proportional cost decrease significantly over time. We also find that the fixed cost is generally not equal to zero. Surprisingly, it goes negative lately.
\end{abstract}

\section{Keywords}

Tick-by-Tick Data, Market Impact Cost, Proportional Cost, Fixed Cost

\section{Introduction}

Over the last decade, trading cost analysis has been one of the biggest areas of investment for both the buy side and sell side of the equity industry, because trading cost can make the implementation of their trading strategies expensive. Implicit trading cost, which reflects the cost of trading due to its impacts upon prices, is one crucial part of trading cost and accounts for a substantial part of the total trading cost. Since implicit trading cost is a function of price impact of a trade, studying this type of cost requires the examination of the relationship between price changes and order flows.

There has been an extensive empirical literature documenting the effects of order flows on the stock price changes. For example, [1] [2] [3] find that asset prices respond positively to order flows and are related to proportional costs multiplied by the signs of order flows (positive for purchases and negative for sales $)^{1}$. More generally speaking, there is a large literature on the relationships ${ }^{1}$ The seminar Kyle model in [4] predicts a positive relationship between price changes and order flows, which is called as market impact cost. See also [5]. 
between trades and price changes, such as [2] [4] [6] [7] [8] [9] [10], among others. However, these studies use small data sets that typically cover only a couple of years of tick-by-tick trades. Therefore, each of them gives a snapshot of implicit trading costs in a short and specific period. We, however, extend the previous studies by using a much larger data set (about 13-year data) ${ }^{2}$. We are able to study how different components of implicit trading costs change over time.

More importantly, we examine the relationship between price changes and changes in the signs of order flows and the relationship between price changes and changes in the inverses of order flows simultaneously. None of the above papers study the relationship between price changes and changes in the inverses of order flows. We model these two relationships as a result of compensating the market maker who incurs an affine structure of trading costs when processing order flows. The costs comprise two components: one is fixed cost, which is constant, and the other is proportional cost, which is proportional to the trade size. Because the market maker is competitive, she earns zero expected profits. As a result, she passes these costs onto the investor in equilibrium.

We use tick-by-tick data of stocks listed on NASDAQ to test the above relationships and study the dynamics of different components of implicit trading costs $^{3}$. We first estimate the market impact cost, fixed cost, and proportional cost for each stock and each quarter. We then perform cross-sectional tests for each quarter. To take account of the fact that the distributions of the estimated costs are not normally distributed, we perform Wilcoxon signed rank test, a non-parametric test, of the hypotheses that the median values of the cross-sectional estimates of the different components of implicit trading costs are equal to zeros. Empirical results reject the above hypotheses and we find that these components are significantly different from zeros. Furthermore, we find that the market impact cost, which may be due to asymmetric information, remains stable over time. The median value of market impact cost is around 0.84 dollars per million shares. However, the fixed cost and the proportional cost, which are due to institutional and technical factors, decrease significantly over time because of institutional innovation and technological advance. The median values are 5.0 dollars and 0.136 dollars at the beginning of 1993. Their values decrease to -0.102 dollars and 0.0102 dollars by the end of 2005. Surprisingly, the fixed cost can even be negative. Since institutional investors tend to submit big orders and retail investors tend to submit small orders, the market impact cost is more important for institutional investors, whereas the trading costs due to the affine structure of ${ }^{2}$ Market makers are responsible for creating and maintaining a market for securities listed on NASDAQ. The tick-by-tick data are the most detailed display of a market's trading information. The Tick-by-tick shows every trade that occurs and provides a variety of information about each trade (e.g. the exact time, the direction, the number of contracts that were traded, etc.). The Tick-by-tick chart allows to view direction of a market's price movement in detail.

${ }^{3}$ Market makers are responsible for creating and maintaining a market for securities listed on NASDAQ. The tick-by-tick data are the most detailed display of a market's trading information. The Tick-by-tick shows every trade that occurs and provides a variety of information about each trade (e.g. the exact time, the direction, the number of contracts that were traded, etc.). The Tick-by-tick chart allows to view direction of a market's price movement in detail. 
costs faced by the market maker is more important for retail investors. We are perhaps the first to document this affine structure of trading costs in the literature.

The rest of this paper is organized as follows. Section 2 develops a simply model of market impact cost, fixed cost, and proportional cost. Section 3 presents the empirical estimation of the pricing function. Section 4 concludes the paper.

\section{Model}

In this section, we extend the Kyle model by including both fixed and proportional costs. In Subsection 2.1, we consider the standard one-shot Kyle equilibrium in which the market maker quotes a linear price schedule that contains only market impact cost. We can solve the full equilibrium in this case. Since institutional constraints such as discreteness and order process costs can also lead to the existence of fixed and proportional costs, we next study a case in which the market maker trades with an affine structure of costs in Subsection 2.2. We assume that there is no information asymmetry. We can derive the full equilibrium in this case as well. In equilibrium the price contains both proportional and fixed costs, which compensate the trading costs incurred by the market makers. In Section 2.3, we study the general case, in which the informed trader optimizes against an exogenous price schedule with market impact cost, proportional cost, and fixed cost. Due to the non-trading region of the informed investor, we are unable to derive the full equilibrium and thus assume an exogenous price schedule.

\subsection{One-Shot Kyle Model}

We consider a standard Kyle model. There are three types of traders: a risk-neutral strategic trader, a competitive and risk-neutral market maker who sets stock prices competitively, and noise traders. There is one risk-free bond and one risky stock available for trading. Without loss of generality, we assume that the interest rate for the bond is zero and that the price of the bond is always 1 . At the end of period one, the game ends and all participants receive payments according to their stock holdings. The terminal payoff of the stock is $v \sim N\left(v_{0}, \sigma_{v}^{2}\right)$, where $v_{0}$ is a positive constant. The informed trader knows $v$ and his demand is $x$. Noise traders submit a net order flow $\mu \sim N\left(0, \sigma_{u}^{2}\right)$, which is independent of $v$. The market maker observes the total demand $y=x+u$. Shen then sets a price $P$ and the market clears. We assume that their initial endowments are zeros.

Since this is a standard model, we omit the proof and summarize the results in the following proposition.

Proposition 1. The price is given by

$$
P=v_{0}+\lambda y
$$

where $v_{0}$ is the unconditional expected value of the stock's terminal payoff, 
representing the market makers' prior, and $\lambda=\frac{\sigma_{v}}{2 \sigma_{u}}$, and the demand of the informed trader is given by

$$
x=x_{0}+\beta v,
$$

where $\beta=\frac{\sigma_{u}}{\sigma_{v}}$ and $x_{0}=\beta v_{0}$.

[4] also considers the dynamic trading in a continuous-time interval $[0,1]$ and shows that $P_{t}=v_{0}+\lambda y_{t}$ still holds, where $y_{t}$ represents the accumulated order flow at time $t$. Therefore, $\Delta P_{t} \equiv P_{t}-P_{t-1}=\lambda \Delta y$, where $\Delta y_{t}$ is the order flow at time $t$ and $\Delta$ is the difference algorithm. The price function implies that price changes are positively related to order flows.

\subsection{Fixed and Proportional Costs}

In reality, institutional factors introduce other types of implicit trading costs other than market impact cost. We assume that the market maker incurs an affine structure of trading costs when processing orders from investors. The cost function is given by $c_{1}+c_{2}|\Delta y|$, where $\Delta y$ is the market order she clears. We term $c_{1}$ as the fixed cost and $c_{2}$ as the proportional cost. To derive a close-form solution, we assume that there is no information asymmetry in the economy and thus no market impact cost. Because the market maker is competitive, she earns zero expected profit. Therefore, she will pass the trading costs on the investors. As a result, the competitive risk-neutral market maker always sets the price equal to $v_{0}$ adjusted for liquidity premia to compensate for the affine structure of trading costs she incurs.

Since the market maker is competitive, the expected profit of the market maker is zero. Therefore, $0=E\left[(p-v) \Delta y-c_{1}-c_{2}|\Delta y|\right]$. Rearrangement yields the price in equilibrium. We summarize the results in the following proposition.

Proposition 2. When the market maker incurs an affine structure of trading costs and there is no information asymmetry, the price is given by

$$
P=v_{0}+\frac{c_{1}}{\Delta y}+c_{2} \operatorname{sign}(\Delta y) .
$$

Since the market maker has to be compensated for the trading costs she incurs when she trades, there exists one component in the price function proportional to the sign of the order flow and there exists one component in the price function proportional to the inverse of the order flow. Therefore, price changes are proportional to changes in the signs of order flows and changes in the inverses of order flows.

\subsection{General Cost Function}

In this subsection, we consider the general implicit cost functions, including fixed cost, proportional cost, and market impact cost. The existence of these trading costs leads to a non-trading region of the informed investor. Therefore, the total order flow is not normally distributed, though the signal the informed 
investor observes and the noise trading are both normally distributed. As a result, a linear pricing scheme is not optimal for the market maker, which prevents a calculation of the full equilibrium. Since it is important to consider the effects of fixed and proportional costs, we assume that the market maker set a price rule given by

$$
P=v_{0}+\lambda y+c_{1} \frac{1}{\Delta y}+c_{2} \operatorname{sign}(\Delta y)
$$

where $\lambda=\frac{\sigma_{v}}{2 \sigma_{u}}$ and $y=\Delta y$. Note that following Kyle (1985), we use $y$ instead of $\Delta y$ for $\lambda^{4}$.

Simple calculation yields that

$$
x= \begin{cases}\beta\left(v-v_{0}\right)-c_{2} \operatorname{sign}\left(v-v_{0}\right) & \text { if }\left|v-v_{0}\right| \geq \bar{v} \\ 0 & \text { if }\left|v-v_{0}\right|<\bar{v}\end{cases}
$$

where $\beta=\frac{\sigma_{u}}{\sigma_{v}}$ and $\bar{v}$ satisfies $(\bar{v}-p) x=0$. Note that because of the existence of the proportional cost and the fixed cost faced by the market maker, the informed trader trades less aggressively compared with the case in Section 2.1.

\section{Empirical Estimation}

Empirically, [1] [2] [3] have discovered that asset prices respond positively to order flows and are related to the sign of trade size. These studies, however, do not test the nonlinear component in the price function associated with the fixed cost in the affine structure of trading costs faced by the market maker. In addition, we explore a larger data set and focus on the dynamics of different components of implicit trading costs over a decade.

We apply [2] method to estimate the market impact cost, fixed cost, and proportional cost. We assume that $v_{0}$ follows a unit root process. Following the explanation in previous section, the price change process is then given by

$$
\Delta P_{t}=\lambda \Delta y_{t}+c_{2}\left(\psi_{t}-\psi_{t-1}\right)+c_{1}\left(\frac{1}{\Delta y_{t}}-\frac{1}{\Delta y_{t-1}}\right)+\epsilon_{t},
$$

where $\Delta P_{t}$ is the price change at transaction $t, \Delta y_{t}$ is the order flow, $\epsilon=v_{0, t}-v_{0, t-1}$, and $\psi_{t}=\operatorname{sign}\left(\Delta y_{t}\right)$, that is, if the trade size $\Delta y_{t}$ is positive, $\psi_{t}=1$, otherwise, $\psi_{t}=-1$.

We estimate the market impact cost $\lambda$ and coefficients $c_{1}$ and $c_{2}$ in the affine structure of trading costs with Equation (5) for each stock and each quarter using tick-by-tick trade data. Given that we have such a massive data set, we apply Ordinary Least Square (OLS) to estimate these cost components. To take into account potential misspecifications, we include an intercept term in the regression specification. Following the convention of [9], for each transaction the quote preceding the transaction by at least 5 seconds is associated with the trade. ${ }^{4}$ When studying the determinants of trade size, Brennan and Subrahmanyam (1998) also assume an exogenous price schedule. 
We classify trades as buyer-or-seller initiated trades as follows. If a transaction occurs above (below) the matched quote mid-points, it is regarded as a purchase (sell). Following [1], if a transaction occurs exactly at the midpoint of the bid and ask, it is signed using the previous transaction price according to the tick test [i.e. buys (sells) if the sign of the last nonzero price change is positive (negative)].

We then perform cross-sectional tests. To take account of the fact that the distributions of the estimated costs are not normally distributed, we perform Wilcoxon signed rank test, a non-parametric test, of the hypotheses that the median values of cross-sectional estimates of different components of implicit trading costs are equal to zeros. More specifically, we test the following hypotheses ${ }^{5}$ :

$$
\text { median } \lambda=0 \text {, median } c_{1}=0 \text {, median } c_{2}=0 \text {. }
$$

\subsection{Data and Descriptive Statistics}

We consider a sample of stocks traded on the NASDAQ ${ }^{6}$. We use tick-by-tick trades contained in the Trades and Automated Quotes (TAQ) database to estimate the cost parameters $\lambda, c_{1}$, and $c_{2}$ for each stock. We follow [11] to determine the sign of a trade. The time period is from January 1, 1993 through December 31, 2005. We use the University of Chicago's Center for Research in Security Prices (CRSP) daily database to determine the relevant stocks for the estimation of the above three types of costs. We estimate our model within each quarter. Following [12], we clean the data and exclude stocks for the following reasons: 1) ADRs and REITs; 2) Closing price below 2 dollars on at least one day; 3) Not traded each day; and 4) Stock split. Thus, this sample consists of all common stocks that had ticker names on both CRSP and TAQ and were traded for a three-month period.

Following [12] [13], quotes from the exchange other than the exchange of listing are excluded, and the price and quote data must occur between 9:30 AM and 4:00 PM. We omit the overnight price changes from the analysis to avoid mixing the price change series with those at the opening. We exclude the price when there is no opening quote. Thus, the first trade after the opening time is ignored. We also exclude the trades with negative prices. In addition, only quotes that satisfy the following conditions are retained: the bid-ask spread is positive and below five dollars, the bid-ask spread divided by the midpoint of the quoted bid and ask is less than $10 \%$ if the midpoint is greater than or equal to 50 dollars, and the quoted spread is less than $25 \%$ for midpoints less than 50 dollars. These conditions guarantee the use of reasonable quotes.

\footnotetext{
${ }^{5}$ Wilcoxon signed rank test is defined as follows. Assume that $x_{i}$ is an observation, where $i=1,2,3, \cdots$. The hypothesis is that the median of $x_{i}$ equals $\mu_{0}$. Wilcoxon rank test statistics is given by $S=\sum r_{i}^{+}-\left(n_{i}+1\right) n_{i} / 4$, where $r_{i}^{+}$is the rank of $\left|x_{i}-\mu_{0}\right|$ after discarding values of $x_{i}$ equal to $\mu_{0}, n_{t}$ is the number of values not equal to $\mu_{0}$, and the sum is calculated for values of $x_{i}-\mu_{0}$ greater than 0 . Average ranks are used for tied values.

${ }^{6}$ We have also considered stocks traded on the NYSE and obtained similar results.
} 
The number of stocks in our sample increased from the first quarter of 1993 and stabilized after 1997. We pool the estimates of market impact cost $\lambda$, proportional cost $c_{1}$, and fixed cost $c_{2}$ for each quarter together ${ }^{7}$. We have 121,505 observations in total. Descriptive statistics are provided in Table 1. The mean value of $\lambda$ is about 8 dollars per million shares, that is, it takes a buy order of 1 million shares to push up the price by 8 dollars. The mean values of $c_{1}$ and $c_{2}$ are 1.67 dollars and 8 cents, respectively. Therefore, the price moves up 1.67 dollars divided by trade size plus 8 cents for a buy trade. It is compelling to note the kurtosises of the components of implicit trading costs have large magnitudes and the differences between the means and the medians are huge. For example, the median value of $\lambda$ is 0.84 dollars per millions shares, which is only one-tenth of the corresponding mean value of $\lambda$. These facts imply that these components are not normally distributed. We formally test the hypotheses that the costs are normally distributed by Kolmogorov-Smirno tests. The second last column of Table 1 shows that normality hypotheses are rejected at $1 \%$ significance level. The magnitudes of the variances and the kurtosises may imply that there are some extreme values in the estimated costs, which may be potential outliers ${ }^{8}$. After examining the descriptive statistics, we next perform cross-sectional tests for each quarter to study the dynamics of the different components of implicit trading costs over time. To take account of the non-normality distribution of the costs and the potential outliers, we use Wilcoxon signed rank test in the following analysis. The last column shows the results of pooling estimation. The signed rank tests show that the median estimates of $\lambda, c_{1}$, and $c_{2}$ are all different from zero at $1 \%$ significance levels. We further examine the dynamics of $\lambda, c_{1}$, and $c_{2}$ over time in the next section.

\subsection{Results}

Tables 2-4 present the median values of market impact cost $\lambda$, proportional cost $c_{1}$, and fixed cost $c_{2}$ for each quarter. They represent the results from 1993 through 1997, from 1998 through 2001, and from 2002 through 2005, respectively.

We first examine market impact cost. Panel A of Figure 1 plots the median $\lambda$ against time $t$. Overall, the market impact cost $\lambda$ is significantly larger than $5 \mathrm{E}-7$ at $1 \%$ significance level, which is consistent with previous studies. The average median $\lambda \mathrm{s}$ for these three periods are $6.34 \mathrm{E}-7,9.67 \mathrm{E}-7$, and $1.02 \mathrm{E}-6 \mathrm{dol}-$ lars, respectively ${ }^{9}$. It shows that the market impact cost remains stable over time, although it increases in the period from 2001 to 2003 and then decreases in the following period. The stability of market impact cost over time is consistent with

${ }^{7}$ Given the massive tick-by-tick data set, we are unable to estimate these costs for each stock for our whole sample.

${ }^{8}$ Since we use tick-by-tick data, which is usually very messy, it is not surprising that we have some outliers.

${ }^{9}$ For example, $\lambda=1.02 \mathrm{E}-6$ means that a trade of 1 million shares moves the price by 1.02 dollars. 
the notion that the source of market impact cost is due to information asymmetry, which may be stable over time.

Table 1. Descriptive statistics of estimated costs, 1993-2005.

\begin{tabular}{cccccccc}
\hline & $N$ & Mean & $\begin{array}{c}\text { Standard } \\
\text { error }\end{array}$ & Skewness & Kurtosis & $\begin{array}{c}\text { Normality } \\
\text { test }\end{array}$ & Median \\
\hline$\lambda$ & 121,505 & 0.79 & 2220 & 314.65 & 103668.82 & $0.49^{*}$ & $0.84^{*}$ \\
$C_{1}$ & 121,505 & 1.67 & 75.90 & 121.93 & 42900.14 & $0.44^{*}$ & $0.396945^{*}$ \\
$C_{2}$ & 121,505 & 0.08 & 0.83 & 94.96 & 41634.46 & $0.45^{*}$ & $0.061983^{*}$ \\
\hline
\end{tabular}

We pool the estimates of the implicit trading costs for each stock and each quarter from 1993 through 2005 together. We test the normalities of the $\lambda, c_{1}$, and $c_{2}$ by Kolmogorov-Smirno test. We perform Wilcoxon signed rank test of the hypotheses that the median estimates are different from zeros. ${ }^{*}$ indicates significance at the $1 \%$ level.

Table 2. Estimates of implicit trading costs, 1993-1997.

\begin{tabular}{|c|c|c|c|}
\hline Quarter & $\lambda$ & $C_{1}$ & $C_{2}$ \\
\hline $93 / 01$ & $0.578^{\star *}$ & $5.000^{*}$ & $0.136^{*}$ \\
\hline $93 / 02$ & $0.619^{*}$ & $5.107^{\star}$ & $0.133^{*}$ \\
\hline $93 / 03$ & $0.618^{*}$ & $5.257^{\star}$ & $0.130^{*}$ \\
\hline $93 / 04$ & $0.645^{\star}$ & $5.065^{\star}$ & $0.129^{*}$ \\
\hline $94 / 01$ & $0.612^{*}$ & $5.100^{*}$ & $0.130^{*}$ \\
\hline $94 / 02$ & $0.658^{*}$ & $4.884^{*}$ & $0.123^{*}$ \\
\hline $94 / 03$ & $0.684^{\star}$ & $4.488^{*}$ & $0.116^{\star}$ \\
\hline $94 / 04$ & $0.623^{*}$ & $3.946^{*}$ & $0.109^{*}$ \\
\hline $95 / 01$ & $0.665^{\star}$ & $3.786^{*}$ & $0.107^{\star}$ \\
\hline $95 / 02$ & $0.616^{*}$ & $3.465^{*}$ & $0.106^{*}$ \\
\hline $95 / 03$ & $0.649^{*}$ & $3.399^{*}$ & $0.111^{\star}$ \\
\hline $95 / 04$ & $0.558^{\star}$ & $3.401^{\star}$ & $0.110^{*}$ \\
\hline $96 / 01$ & $0.562^{*}$ & $3.270^{*}$ & $0.113^{*}$ \\
\hline $96 / 02$ & $0.588^{*}$ & $2.900^{*}$ & $0.114^{*}$ \\
\hline $96 / 03$ & $0.696^{*}$ & $3.020^{*}$ & $0.109^{*}$ \\
\hline $96 / 04$ & $0.613^{*}$ & $2.740^{\star}$ & $0.104^{\star}$ \\
\hline $97 / 01$ & $0.595^{\star}$ & $2.806^{*}$ & $0.100^{*}$ \\
\hline $97 / 02$ & $0.702^{\star}$ & $2.551^{\star}$ & $0.093^{*}$ \\
\hline $97 / 03$ & $0.699^{*}$ & $1.937^{\star}$ & $0.086^{*}$ \\
\hline $97 / 04$ & $0.701^{\star}$ & $1.632^{*}$ & $0.080^{*}$ \\
\hline
\end{tabular}

$$
\Delta P_{t}=\lambda \Delta y_{t}+A_{M}\left(v_{t}-v_{t-1}\right)+B_{M} / \Delta y_{t}-B_{M} / \Delta y_{t-1}+\epsilon_{t},
$$

where $\Delta P_{t}$ is the price change at transaction $t, \Delta y_{t}$ is the order flow, $\epsilon=v_{0, t}-v_{0, t-1}$, and $\psi_{t}=\operatorname{sign}\left(\Delta y_{t}\right)$. Thus, if the signed trade size $\Delta y_{t}$ is positive, $v_{t}=1$, otherwise, $v_{t}=-1$. Here, we assume that $\epsilon_{t}$ is identically and independently distributed. We perform Wilcoxon signed rank test of the hypotheses that the median estimates are different from zeros. ${ }^{*}$ indicates significance at the $1 \%$ level. 
Table 3. Estimates of implicit trading costs.

\begin{tabular}{cccc}
\hline Quarter & $\lambda$ & $c_{1}$ & $c_{2}$ \\
\hline $98 / 01$ & $0.697^{*}$ & $1.307^{*}$ & $0.071^{*}$ \\
$98 / 02$ & $0.703^{*}$ & $1.040^{*}$ & $0.067^{*}$ \\
$98 / 03$ & $0.682^{*}$ & $0.793^{*}$ & $0.073^{*}$ \\
$98 / 04$ & $0.602^{*}$ & $0.494^{*}$ & $0.073^{*}$ \\
$99 / 01$ & $0.689^{*}$ & $0.352^{*}$ & $0.070^{*}$ \\
$99 / 02$ & $0.818^{*}$ & $0.244^{*}$ & $0.067^{*}$ \\
$99 / 03$ & $0.939^{*}$ & $0.064^{*}$ & $0.062^{*}$ \\
$99 / 04$ & $0.980^{*}$ & $0.079^{*}$ & $0.067^{*}$ \\
$00 / 01$ & $1.00^{*}$ & $0.123^{*}$ & $0.081^{*}$ \\
$00 / 02$ & $1.16^{*}$ & $0.019^{*}$ & $0.074^{*}$ \\
$00 / 03$ & $1.23^{*}$ & $-0.215^{*}$ & $0.060^{*}$ \\
$00 / 04$ & $1.00^{*}$ & $-0.200^{*}$ & $0.063^{*}$ \\
$01 / 01$ & $1.24^{*}$ & $-0.366^{*}$ & $0.052^{*}$ \\
$01 / 02$ & $1.31^{*}$ & $-0.426^{*}$ & $0.033^{*}$ \\
$01 / 03$ & $1.15^{*}$ & $-0.196^{*}$ & $0.038^{*}$ \\
$01 / 04$ & $1.31^{*}$ & $-0.142^{*}$ & $0.034^{*}$ \\
\hline & $\Delta P_{t}=\lambda \Delta y_{t}+A_{M}\left(v_{t}-v_{t-1}\right)+B_{M} / \Delta y_{t}-B_{M} / \Delta y_{t-1}+\epsilon_{t}$, & \\
\hline
\end{tabular}

where $\Delta P_{t}$ is the price change at transaction $t, \Delta y_{t}$ is the order flow, $\epsilon=v_{0, t}-v_{0, t-1}$, and $\psi_{t}=\operatorname{sign}\left(\Delta y_{t}\right)$. Thus, if the signed trade size $\Delta y_{t}$ is positive, $v_{t}=1$, otherwise, $v_{t}=-1$. Here, we assume that $\epsilon_{t}$ is identically and independently distributed. We perform Wilcoxon signed rank test of the hypotheses that the median estimates are different from zeros. * indicates significance at the $1 \%$ level.

Table 4. Estimates of implicit trading costs, 2002-2005.

\begin{tabular}{|c|c|c|c|}
\hline Quarter & $\lambda$ & $c_{1}$ & $C_{2}$ \\
\hline $02 / 01$ & $1.22^{*}$ & $-0.152^{*}$ & $0.029^{*}$ \\
\hline $02 / 02$ & $1.31^{*}$ & $-0.123^{*}$ & $0.028^{*}$ \\
\hline $02 / 03$ & $1.31^{*}$ & $-0.096^{*}$ & $0.028^{*}$ \\
\hline $02 / 04$ & $1.11^{*}$ & $-0.141^{*}$ & $0.020^{*}$ \\
\hline $03 / 01$ & $1.07^{*}$ & $-0.073^{*}$ & $0.014^{*}$ \\
\hline $03 / 02$ & $1.01^{*}$ & $-0.087^{\star}$ & $0.015^{\star}$ \\
\hline $03 / 03$ & $1.01^{*}$ & $-0.072^{*}$ & $0.015^{*}$ \\
\hline $03 / 04$ & $0.94^{*}$ & $-0.098^{*}$ & $0.015^{*}$ \\
\hline $04 / 01$ & $0.90^{*}$ & $-0.125^{*}$ & $0.014^{\star}$ \\
\hline $04 / 02$ & $1.08^{*}$ & $-0.137^{*}$ & $0.013^{*}$ \\
\hline $04 / 03$ & $1.07^{*}$ & $-0.139^{*}$ & $0.012^{*}$ \\
\hline $04 / 04$ & $0.91^{*}$ & $-0.137^{\star}$ & $0.014^{\star}$ \\
\hline $05 / 01$ & $0.92^{*}$ & $-0.084^{*}$ & $0.013^{*}$ \\
\hline $05 / 02$ & $0.84^{*}$ & $-0.097^{\star}$ & $0.012^{*}$ \\
\hline $05 / 03$ & $0.85^{\star}$ & $-0.097^{\star}$ & $0.013^{\star}$ \\
\hline 05/04 & $0.77^{*}$ & $-0.103^{*}$ & $0.012^{*}$ \\
\hline
\end{tabular}

$$
\Delta P_{t}=\lambda \Delta y_{t}+A_{M}\left(v_{t}-v_{t-1}\right)+B_{M} / \Delta y_{t}-B_{M} / \Delta y_{t-1}+\epsilon_{t},
$$

where $\Delta P_{t}$ is the price change at transaction $t, \Delta y_{t}$ is the order flow, $\epsilon=v_{0, t}-v_{0, t-1}$, and $\psi_{t}=\operatorname{sign}\left(\Delta y_{t}\right)$. Thus, if the signed trade size $\Delta y_{t}$ is positive, $v_{t}=1$, otherwise, $v_{t}=-1$. Here, we assume that $\epsilon_{t}$ is identically and independently distributed. We perform Wilcoxon signed rank test of the hypotheses that the median estimates are different from zeros. ${ }^{*}$ indicates significance at the $1 \%$ level. 
We next examine the affine structure of trading costs. The average median fixed costs in these three periods are 3.69 dollars, 0.19 dollars, and -0.11 dollars, respectively. The average median proportional costs for these periods are 11 cents, 6 cents, and 2 cents, respectively ${ }^{10}$. Overall, this affine structure of implicit trading costs, including both the fixed cost and the proportional cost, drop dramatically over time. Panel B and panel C of Figure 1 plot the median estimates of $c_{1}$ and $c_{2}$ against time $t$, respectively. It is clear that the median estimate of $c_{1}$ decreases from around 5 dollars per trade at the beginning of 1993 to -0.11 dollars per trade by the end of 2005 and the median estimate of $c_{2}$ decreases from 14 cents per share per trade to 2 cents per share per trade. Note the fixed component in the affine structure of trading costs can be negative. It means that the market maker pays the investor who trades with a lum-sum money. However, since the proportional cost is always positive, the market still gets compensation for providing liquidity service. Since the affine structure of trading costs are more closely related to the institutional factors, such as price discreteness and the order processing cost faced by the market maker, institutional innovation and technological advance may explain this declining time trend. It is interesting to compare the effects of the three components of implicit trading costs on investors. We assume that $\lambda=1.02 \mathrm{E}-6$ dollars, $c_{1}=-0.11$ dollars, and $c_{2}=2$ cents. If an investor submits a big order of 100,000 shares, the market impact cost and the cost due to the affine structure of costs faced by the market marker are 10,200 dollars and 1999.89 dollars, respectively. However, If an investor submits a small order of 1000 shares, the market impact cost and the cost due

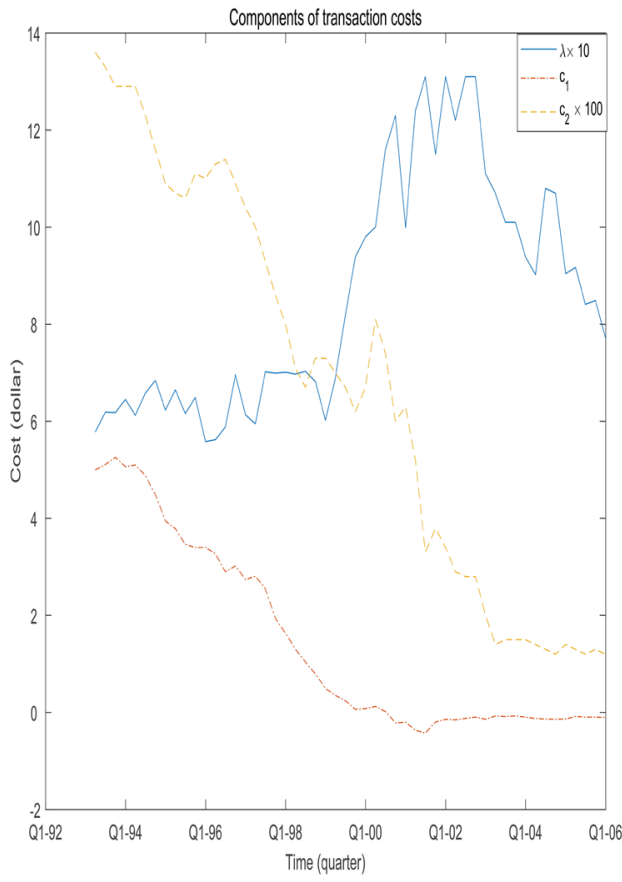

Figure 1. Median trading costs plot against time: 1993-2005.

${ }^{10}$ For example, $c_{1}=0.19$ and $c_{2}=0.06$ mean that the market maker will be compensated with 0.19 dollars plus 2 cents multiplied by the trade size per trade. 
to the affine structure of costs faced by the market marker are 1.02 dollars and 19.89 dollars, respectively. Since institutional investors tend to submit big orders and retail investors tend to submit small orders, the market impact cost is more important for institutional investors, whereas the trading costs due to the affine structure of costs faced by the market maker is more important for retail investors.

In summary, our empirical study shows a nonlinear stock price function. The market impact cost, which may be due to asymmetric information, remains stable over time, but the fixed cost and the proportional cost components, which are due to institutional factors, decrease significantly over time because of technology and institutional innovations.

\section{Conclusion}

We use tick-by-tick trade data to examine the dynamics of different components of implicit trading costs over a decade, including market impact cost, fixed cost, and proportional cost. We model the fixed cost and the proportional cost as an affine structure of trading costs faced by the market maker. We find that the market impact cost remains stable over time, but the fixed cost and the proportional cost decrease significantly over time, which may be explained by technology and institutional innovations. We find that the fixed cost is generally not equal to zero. Surprisingly, it goes negative lately.

\section{Acknowledgements}

I thank Pete Kyle, Hui Ou-Yang, and seminar participants at ShanghaiTech and Peking for comments. This paper is supported by National Natural Science Foundation of China (No. 71773077).

\section{Conflicts of Interest}

The author declares no conflicts of interest regarding the publication of this paper.

\section{References}

[1] Brennan, M.J. and Subrahmanyam, A. (1998) The Determinant of Average Trade size. Journal of Business, 71, 1-25. https://doi.org/10.1086/209734

[2] Glosten, L. and Harris, L. (1988) Estimating the Components of the Bid-Ask Spread. Journal of Financial Economics, 21, 123-142. https://doi.org/10.1016/0304-405X(88)90034-7

[3] Hasbrouck, J. (1991) Measuring the Information Content of Stock Trades. Journal of Finance, 46, 179-207. https://doi.org/10.1111/j.1540-6261.1991.tb03749.x

[4] Kyle, A.S. (1985) Continuous Auctions and Insider Trading, Econometrica, 53, 1315-1336. https://doi.org/10.2307/1913210

[5] Easley, D. and O'Hara, M. (1987) Price, Trade Size, and Information in Securities Markets. Journal of Financial Economics, 19, 69-90. https://doi.org/10.1016/0304-405X(87)90029-8 
[6] Huang, R. and Stoll, H. (1997) The Components of the Bid-Ask Spread: A General Approach. Review of Financial Studies, 10, 995-1034. https://doi.org/10.1093/rfs/10.4.995

[7] Madhavan, A., Richardson, M. and Roomans, M. (1997) Why Do Security Prices Change? A Transaction-Level Analysis of NYSE Stocks. Review of Financial Studies, 10, 1035-1064. https://doi.org/10.1093/rfs/10.4.1035

[8] Manganelli, S. (2005) Duration, Volume and Volatility Impact of Trades. Journal of Financial Markets, 8, 377-399. https://doi.org/10.1016/j.finmar.2005.06.002

[9] Roll, R. (1984) A Simple Implicit Measure of the Effective Bid-Ask Spread in an Efficient Market. Journal of Finance, 39, 1127-1139. https://doi.org/10.1111/j.1540-6261.1984.tb03897.x

[10] Weber, P. and Rosenow, B. (2006) Large Stock Price Changes: Volume or Liquidity? Quantitative Finance, 6, 7-14. https://doi.org/10.1080/14697680500168008

[11] Lee, C. and Ready, M. (1991) Inferring Trade Direction from Intradaily Data. Journal of Finance, 46, 733-746. https://doi.org/10.1111/j.1540-6261.1991.tb02683.x

[12] Stoll, H.R. (2000) Friction. Journal of Finance, 55, 1479-1514. https://doi.org/10.1111/0022-1082.00259

[13] Chordia, T., R. Roll and Subrahmanyam, A. (2001) Market Liquidity and Trading Activity. Journal of Finance, 56, 501-530. https://doi.org/10.1111/0022-1082.00335 